\title{
SRRM4 Expands the Repertoire of Circular RNAs by Regulating Microexon Inclusion
}

\author{
Vanessa M. Conn ${ }^{1,2}$, Marta Gabryelska ${ }^{1,2} \mathbb{D}$, Shashikanth Marri ${ }^{1,2}$, Brett W. Stringer 1,2,3 (D), \\ Rebecca J. Ormsby 1,2 , Timothy Penn ${ }^{1,2}$, Santosh Poonnoose ${ }^{2,4}$, Ganessan Kichenadasse ${ }^{2,5}$ \\ and Simon J. Conn 1,2,*(D)
}

1 Flinders Cancer Research, College of Medicine and Public Health, Flinders University, Bedford Park 5042, South Australia, Australia; vanessa.conn@flinders.edu.au (V.M.C.); marta.gabryelska@flinders.edu.au (M.G.); shashikanth.marri@flinders.edu.au (S.M.); brett.stringer@flinders.edu.au (B.W.S.); rebecca.ormsby@flinders.edu.au (R.J.O.); timothy.penn@flinders.edu.au (T.P.)

2 Flinders Health and Medical Research Institute (FHMRI), College of Medicine and Public Health, Flinders University, Bedford Park 5042, South Australia, Australia; santosh.poonnoose@flinders.edu.au (S.P.); ganessan.kichenadasse@flinders.edu.au (G.K.)

3 School of Clinical Medicine-Greenslopes Clinical Unit, The University of Queensland, Brisbane 4120, Queensland, Australia

4 Department of Neurosurgery, Flinders Medical Centre, Bedford Park 5042, South Australia, Australia

5 Department of Medical Oncology, Flinders Centre for Innovation in Cancer, Flinders Medical Centre, Bedford Park 5042, South Australia, Australia

* Correspondence: simon.conn@flinders.edu.au; Tel.: +61-8-8404-2816

Received: 6 October 2020; Accepted: 13 November 2020; Published: 16 November 2020

\begin{abstract}
High-throughput RNA sequencing (RNA-seq) and dedicated bioinformatics pipelines have synergized to identify an expansive repertoire of unique circular RNAs (circRNAs), exceeding 100,000 variants. While the vast majority of these circRNAs comprise canonical exonic and intronic sequences, microexons (MEs) - which occur in 30\% of functional mRNA transcripts-have been entirely overlooked. CircRNAs which contain these known MEs (ME-circRNAs) could be identified with commonly utilized circRNA prediction pipelines, CIRCexplorer2 and CIRI2, but were not previously recognized as ME-circRNAs. In addition, when employing a bespoke bioinformatics pipeline for identifying RNA chimeras, called Hyb, we could also identify over 2000 ME-circRNAs which contain novel MEs at their backsplice junctions, that are uncalled by either CIRCexplorer2 or CIRI2. Analysis of circRNA-seq datasets from gliomas of varying clinical grades compared with matched control tissue has shown circRNAs have potential as prognostic markers for stratifying tumor from healthy tissue. Furthermore, the abundance of microexon-containing circRNAs (ME-circRNAs) between tumor and normal tissues is correlated with the expression of a splicing associated factor, Serine/arginine repetitive matrix 4 (SRRM4). Overexpressing SRRM4, known for regulating ME inclusion in mRNAs critical for neural differentiation, in human HEK293 cells resulted in the biogenesis of over 2000 novel ME-circRNAs, including ME-circEIF4G3, and changes in the abundance of many canonical circRNAs, including circSETDB2 and circLRBA. This shows SRRM4, in which its expression is correlated with poor prognosis in gliomas, acts as a bona fide circRNA biogenesis factor. Given the known roles of MEs and circRNAs in oncogenesis, the identification of these previously unrecognized ME-circRNAs further increases the complexity and functional purview of this non-coding RNA family.
\end{abstract}

Keywords: circular RNAs; alternative splicing; splicing factors; microexons; SRRM4; glioblastoma 


\section{Introduction}

In its simplest terms, RNA splicing converts precursor mRNA into mature mRNA through the removal of introns and sequential ligation of canonical exons. The RNA splicing machinery, called the spliceosome, is a large and dynamic ribonucleoprotein complex consisting of small nuclear RNAs and approximately 80 distinct proteins [1,2]. Like any enzymatic process, RNA splicing is affected by the accessibility and structure of its substrate, in this case, the $5^{\prime}$ and $3^{\prime}$ ends of an intron by scanning for the canonical GU and AG splice sites, respectively. However, non-canonical RNA transcripts are frequently made through a process called alternative splicing as a result of many factors including RNA secondary structure, strength of splice recognition sites and dynamic permutations of RNA and protein factors within the spliceosome [3,4]. It is estimated that 90-95\% of all eukaryotic genes are alternatively spliced, and this represents the greatest source of expansion of the functional transcriptome and proteome.

Alternative splicing is a dynamic, well-orchestrated, and cell-type specific process which play critical roles in cell differentiation, maintenance of stem cell pluripotency [5] and generation of highly tissue-specific proteins, particularly in the brain which has the highest rate of alternative splicing in the human body [6]. Perhaps unsurprisingly, misregulation of alternative splicing through mutations of RNA splicing factors and RNA splice sites can be devastating, with evidence of aberrant splicing found in cancer and in neurological diseases [7].

The most common alternative splicing events in humans are exon skipping/inclusion ( $>40 \%$ of variants), use of alternative splice sites, and intron retention. While the size of exons and their flanking introns can impact the profile of alternatively spliced transcripts, in the central nervous system, it has been reported that the inclusion of short exons is more frequent [8,9]. The most highly conserved component of neuronal alternative splicing across development are extremely short exons, known as microexons (MEs). Herein, we utilize the commonly employed definition of MEs as those exons 3-30 nucleotides (nt) in length $[3,10]$.

MEs were originally found in 1985 as two 5 nt exons in the Drosophila homeotic Ubx gene. While found sporadically since that time, it was not until 2003 through a combination of bespoke computational analysis on a reference databases of full-length mRNA sequences, that MEs were systematically identified in a range of model species, including humans [11]. At this time, 223 MEs were identified ( $\sim 1.6 \%$ of human genes), yet more contemporary estimates with the aid of high-throughput RNA sequencing have expanded these numbers dramatically to 2008 (in 1587 genes) [12] and over 13,000 for exon sizes up to $51 \mathrm{nt}$ [13].

While still underappreciated, a focus of research into MEs has been in neural development and disease [14]. Although there exists a number of neural-specific RNA splicing factors which affect alternative splicing, including RBFOX1, SRSF11, and PTBP1/2, most neural MEs are regulated by the Serine/arginine repetitive matrix 4 or Neural-specific serine/arginine repetitive splicing factor of $100 \mathrm{kDa}$ (SRRM4/nSR100), through its binding to adjacent intronic enhancer motifs [15,16]. SRRM4 is specifically expressed in the brain and sensory organs and is highly conserved across vertebrates, but lacking in invertebrates, perhaps associated with the increased functional complexity of the vertebrate nervous system [17].

Reinforcing SRRM4 as a master regulator of alternative splicing in the brain, it has been shown to regulate conserved networks of alternative splicing events in human and mouse neural functions including cytoskeletal organization and GTPase signaling [14]. One critical event mediated by SRRM4 is the increased expression of another neural-specific splicing factor, Ptbp2, by regulating the inclusion of exon 10 to prevent nonsense mediated decay driven turnover of Ptbp2 mRNA [17]. However, the most widely known manifestation of SRRM4-regulated alternative splicing is the inclusion of exon 4 in the mRNA of the REST/NSRF transcriptional repressor [18]. This REST/NSRF variant, called REST4, encodes an inactive protein lacking five critical zinc finger DNA binding domains [19,20]. This relieves the transcriptional silencing of a subset of transcripts, allowing neuronal maturation and differentiation to proceed. Therefore, alternative splicing and MEs mediated by SRRM4 are critical in the brain. 
Like MEs, circular RNAs (circRNAs) were originally largely dismissed as outliers of alternative splicing from their initial visual identification in the 1970s [21], and their sequence-based identification in the 1990s [22]. However, with the synthesis of high-throughput RNA sequencing and custom bioinformatics pipelines, circRNAs have been identified as the most contemporary class of alternatively spliced, largely non-coding RNAs, with current estimates of over 100,000 unique circRNAs within the human transcriptome $[23,24]$.

CircRNAs are formed co-transcriptionally and their biogenesis is in competition with canonical linear RNA splicing (Figure 1a) [25]. CircRNA biogenesis is regulated primarily by two non-mutually exclusive mechanisms: (1) the presence of inverted, complementary repeats in flanking introns; and (2) interaction with RNA binding proteins to promote (QKI, FUS, MBNL) or inhibit (ADAR1, mediating adenine to inosine editing) production [23,24,26,27]. In addition, the rate of RNA polymerase II elongation and flanking intron size has also been shown to be positively correlated with circRNA formation from distinct loci $[28,29]$.

It has emerged that alternative splicing is a critical factor in the initiation and progression of human pathologies, including cancer [30,31]. Mutation, or mis-regulation, of splicing factors and snRNAs [32] have been found in various cancers, including the most lethal brain cancer, glioblastoma (GBM). Patients diagnosed with GBM have, on average, 15 months post-diagnosis survival and a 5 -year survival rate below $10 \%$. Standard therapy involves maximal, yet incomplete, surgical resection in combination with chemotherapy (Temozolomide) and/or radiotherapy. However, despite advances in all of these areas, the survival from GBM has increased only $1 \%$ in the past 30 years, compared with $20 \%$ for all other cancers [33]. Alternative splicing signatures have been proposed as a novel prognostic marker, but also targeting alternative splicing in GBM could represent a novel and desperately needed therapeutic strategy [34,35]. CircRNAs have been systematically identified in GBM, with a number of examples of circRNA candidates contributing to hallmarks of the disease [36]. However, further investigation into the fundamental basis of alternative splicing in GBM and profiling circRNAs at various stages of progression of GBM is required.

A number of protein factors have been identified that contribute to circRNA biogenesis and a variety of circRNAs have been identified containing permutations of canonical exons [37]; yet there has been little evidence for the inclusion of unique exons in circRNAs as a result of these protein splicing factors. While upregulation of SRRM4 has been reported to promote neuroendocrine prostate cancer through alternative splicing [38,39], SRRM4 is seen to decrease in expression within the tumor proper compared with the leading edge and infiltrating tumor in GBM based on RNA sequencing data from the IVY Glioblastoma Atlas Project [40]. However, its potential role in brain cancer still remains largely unexplored. Here, to our knowledge, we provide the first report of MEs in circRNAs and extend this by identifying SRRM4 as a master regulator of ME inclusion in GBM and as a bona fide circRNA biogenesis factor.

\section{Materials and Methods}

\subsection{Human Ethics Approval and Patient Tissue Samples}

For all primary tissue sourced for these experiments, written informed consent was obtained from each subject or from their guardian. Specimens were received from the SA Neurological Tumor Bank (SANTB) which is supported by Flinders University, Flinders Foundation and The Neurosurgical Research Foundation. The ethics for this project is approved by the Central Adelaide Local Health Network Human Research Ethics Committee (CALHN HREC) with approval number HREC/17/RAH/284. The SANTB is approved by the Southern Adelaide Clinical Human Research Ethics Committee (SAC HREC) with approval number 286.10. Resected tumor tissue was processed in theatre, placed into sterile cryovials and immediately snap-frozen in liquid nitrogen to preserve RNA integrity and histology. Any non-pathological (normal) brain tissue that was removed during surgery, in order to access the tumor, was collected and processed in a similar manner. Clinical grading was 
performed by SA Pathology and classified according to the WHO classification of tumors of the central nervous system [41].

\subsection{Circular RNA (circRNA) Sequencing}

CircRNA sequencing was performed on 20 tissue samples collected from the South Australian Neurological Tumor Bank with informed patient consent. This included 15 primary gliomas, with five grade II, five grade III, and five grade IV (GBM) tumors as determined by histopathological grading [41] and 5 healthy brain tissue samples (hereafter referred to as healthy, or control tissue; Table 1).

RNA was harvested $48 \mathrm{~h}$ post-transfection using TRIzol ${ }^{\mathrm{TM}}$ (ThermoFisher Scientific, USA) \& Direct-zol ${ }^{\mathrm{TM}}$ RNA miniprep kit (Zymo Research, USA) with on-column DNase I treatment. Ten micrograms of RNA were digested with 20 U Ribonuclease R (Lucigen, USA) at $37{ }^{\circ} \mathrm{C}$ for $30 \mathrm{~min}$. RNA was subsequently purified by RNA MinElute columns (Qiagen, USA) as per Conn et al. [26]. RNA-seq libraries were prepared using Illumina Truseq Stranded Library Preparation Kit with Ribozero Gold rRNA depletion (Illumina, USA). Raw and processed data was deposited in GEO database for HEK293 SRRM4 overexpression data (accession number GSE148813) and glioma circRNA-seq data (accession number GSE159260).

\subsection{CircRNA Prediction}

Reads were mapped against the human reference genome (hg19) using the STAR spliced alignment algorithm [42] (version 2.5.3a with default parameters and-chimSegmentMin 20) returning an average unique alignment rate of $79 \%$ and $81 \%$ for total RNA-seq and circRNA-seq, respectively (Table 1). The resulting STAR produced Chimeric.out.junction file for each sample was parsed and annotated for circRNA prediction and backsplice abundance using CIRCexplorer2 [37]. CIRI2 was used to identify the back-spliced junction reads with default parameters [43]. Bedtools were used to find overlapping microexons in circRNAs based on the genomic coordinate locations [44]. In parallel, reads were processed through the Hyb pipeline [45] utilizing the reference Exon-Intron database from Shepelev et al. [46] and adapted to fit the Hyb-preferred database format by incorporating fragment length and retaining strand information. This database was prepared for Hyb as per Travis et al. [45] The Hyb output format included information about exon number, length, start/end positions in the read and start/end positions in the reference sequence. Junction sites representing circRNAs were established as reads where the end of a downstream exon is followed by beginning of the same or upstream exon.

\subsection{ME-CircRNA Prediction}

For identifying ME-circRNAs, we utilized the known MEs present in linear RNAs from Irimia et al. [15]. Firstly, we assessed if these known MEs were expressed in our data by assessing if reads from the fastq file could be found crossing the ME and at least one flanking exon of the same gene. This was then identified as a ME-circRNA if this ME overlapped a circRNA called by the same pipeline on the same strand. If the ME comprised either the $5^{\prime}$ or $3^{\prime}$ exon of the circRNA, it was called as the backsplice junction, whereas, if it was within the circRNA coordinates but not at either termini, it was referred to as being in the body of the circRNA. This was performed for CIRCexplorer2, CIRI2, and Hyb pipelines.

To identify novel ME-circRNAs, at the beginning of the Hyb analysis we permitted reads of 3-30 nt in-between all chimeric reads. Once identified, the chimeric reads identified as backspliced circRNAs were extracted and filtered to determine microexon length (if present) and sequence for further mapping and analysis of these ME-circRNAs. Calculations of circRNA counts was performed using the merging program within the Hyb package. Format of Hyb-identified circRNAs was then transformed into the more widely used output format of CIRCexplorer2 to contain information on chromosome location, gene name, count, and included exons. This format was used to compare the results to CircExplorer2 and CIRI2. The Hyb package is available at Github (https://github.com/gkudla/hyb), and codes can be provided upon reasonable request. 
Table 1. Sample ID, tumor type and grade, RNA sequencing read and alignment statistics. Tumor sample ID \#267 (colored in red) was excluded from RNA seq analysis due to low percentage mapped reads.

\begin{tabular}{ccccc}
\hline Sample ID & Tumor Type & Grade & \# Input Reads & $\begin{array}{c}\text { \# Uniquely } \\
\text { Mapped Reads }\end{array}$ \\
\hline 158 & Astrocytoma & II & $26,343,021$ & $89.1 \%$ \\
190 & Oligodendroglioma & II & $27,302,075$ & $88.8 \%$ \\
217 & Oligodendroglioma & II & $25,241,413$ & $84.9 \%$ \\
207 & Diffuse glioma & II & $23,492,240$ & $88.4 \%$ \\
267 & Oligodendroglioma & II & $15,437,385$ & $39.1 \%$ \\
150 & Anaplastic astrocytoma & III & $21,617,416$ & $86.6 \%$ \\
154 & Anaplastic oligodendroglioma & III & $29,164,440$ & $90.8 \%$ \\
172 & Anaplastic oligodendroglioma & III & $42,012,355$ & $66.5 \%$ \\
213 & Anaplastic astrocytoma & III & $25,758,766$ & $87.6 \%$ \\
214 & Anaplastic astrocytoma & III & $28,412,184$ & $85.7 \%$ \\
169 & Glioblastoma & IV & $28,268,617$ & $87.6 \%$ \\
170 & Glioblastoma & IV & $27,580,126$ & $82.7 \%$ \\
203 & Glioblastoma & IV & $27,106,465$ & $88.4 \%$ \\
61 & Glioblastoma & IV & $29,197,512$ & $83.0 \%$ \\
160 & Glioblastoma & IV & $23,140,292$ & $86.4 \%$ \\
179 & Control & & $22,852,102$ & $80.3 \%$ \\
61_MC_NOR & Matched Control & & $29,806,403$ & $87.3 \%$ \\
158_MC_NOR & Matched Control & & $28,019,827$ & $88.2 \%$ \\
170_MC_NOR & Matched Control & & $26,038,920$ & $89.1 \%$ \\
267_MC_NOR & Matched Control & & $24,372,321$ & $73.3 \%$ \\
\hline
\end{tabular}

\subsection{Differential Expression Analysis}

Prior to differential expression analysis, circRNAs with very low counts across all the libraries were removed. Low count filtering was done using CPM (Counts Per Million) values rather than counts in order to avoid preference to samples with larger library sizes. Using the edgeR package [47], we calculated median of library sizes and kept genes that have CPM of $\geq 0.01$ in at least three samples for statistical evidence without loss of information [48].

The filtered read counts were normalized using DESeq2 median of ratios [49] method and relative $\log$ transformation [50] utilized to generate the PCA plots. For the differential expression, DESeq2 [51] statistical package was used to assess differential gene expression of circRNAs with Log2fold $>1$ and $p$-adjusted $<0.01$. Volcano plots were generated with EnhancedVolcano R package [52] and heatmaps generated using normalized counts in R. For the circRNAs found in common between all methods, the log-transformed normalized values (CPM) were extracted and plotted on the heatmap using Pearson correlation distance and average linkage method for clustering columns and rows [53].

\subsection{Cell Culture}

HEK293 cells were cultivated at $37{ }^{\circ} \mathrm{C}$ with $5 \% \mathrm{CO}_{2}$ in air in DMEM (Sigma-Aldrich, USA) supplemented with 10\% v/v FBS (Bovogen, Australia) and $1 \mathrm{mg} / \mathrm{mL}$ Antibiotic-Antimycotic (Sigma-Aldrich, USA). Cells were passaged with TrypLE express (ThermoFisher Scientific, USA).

\subsection{Cloning Overexpression Constructs}

Full-length SRRM3 (HsCD00813375) and SRRM4 (HsCD00295084) in pENTR223.1 were obtained from DNASU Plasmid Repository. The coding sequence of each gene, lacking the termination codon, was amplified from this vector using Phusion DNA polymerase (New England Biolabs, USA). The PCR products and pcDNA3.1::FLAG [26] were digested with EcoRI and EcoRV (New England Biolabs, USA), ligated, and transformed into TOP10 chemically competent $E$. coli. Sanger sequencing was performed to validate the overexpression construct. Overexpression of $M E$-circEIF4G3 was performed by using 800nt, reverse complementary introns flanking the circRNA exons (Exons 6a-10) and cloned 
into pcDNA3.1::FLAG as per [26,54]. Sanger sequencing was performed to validate the overexpression construct. HEK293 cells were transiently transfected with expression constructs using Lipofectamine 2000 (ThermoFisher Scientific, USA) in 6-well plates (Sarstedt, Australia).

\section{8. $R T-P C R / q R T-P C R$}

RNA, either total RNA or RNase R-treated RNA, harvested using TRIzol ${ }^{\mathrm{TM}}$ (ThermoFisher Scientific, USA) \& Direct-zol ${ }^{\mathrm{TM}}$ RNA miniprep kit (Zymo Research, USA) with on-column DNase I treatment was reverse transcribed with QuantiTect ${ }^{\circledR}$ reverse transcription kit (Qiagen). After RT, QuantiTect SYBR ${ }^{\circledR}$ Green PCR Kit (Qiagen, USA) was used for qRT-PCR as per [55]. RNA was quantified by NanoDrop One Microvolume UV-Vis Spectrophotometer (ThermoFisher Scientific, USA). All oligonucleotides used in this study were ordered from Integrated DNA Technologies (Singapore), with sequences provided (Table S1).

\subsection{Western Blotting}

Total soluble protein was harvested from HEK293 cells using RIPA buffer with $1 \times$ protease inhibitor cocktails (mini-EDTA free) and phosphatase inhibitor cocktails (ThermoFisher Scientific, USA) with western blotting performed as per Conn et al. [26]. Anti-FLAG M2 antibody (F1804; Sigma-Aldrich USA) was used at 1:2,500 dilution; with goat anti-mouse HRP conjugated secondary antibody (ThermoFisher Scientific, USA) at 1:10,000 dilution. Chemiluminescent detection was carried out using Super Signal West Pico PLUS (ThermoFisher Scientific, USA) and Precision Plus Protein ${ }^{\mathrm{TM}}$ Kaleidoscope ${ }^{\mathrm{TM}}$ Prestained Protein Standard (Bio-Rad, USA) was used for size estimation.

\section{Results}

\subsection{Circular RNA Sequencing across Human Glioma Grades and Control Brain Tissue}

Purified RNA from healthy and glioma tissues underwent digestion with Ribonuclease R (RNase R) to degrade linear RNAs and enrich for circRNAs. Validation of this digestion was achieved by performing RT-PCR on cDNA from RNase R-digested, or mock-treated RNA. In each case, the linear RNA targets were depleted following RNase R digestion, while known circRNA targets could be amplified (Figure S1a). The RNA was depleted for ribosomal RNA and RNA-seq libraries were prepared with the Truseq Stranded Total RNA kit and sequenced by NextSeq 550 using the High output 300 cycle kit, 150PE (Illumina, USA). CircRNA-seq libraries had an average read depth of 27 million reads, with $85 \%$ mapped reads to human genome assemble hg19. One library (Sample \#267, grade II glioma) was excluded due to low percentage of mapped reads $(39.1 \%)$ and significantly distinct circRNA profile from principal component analysis (PCA) on normalized circRNA counts (Figure S1c). Therefore, all further analyses were performed on the remaining 19 samples (Table 1).

CircRNAs were predicted from these libraries using three distinct pipelines, CIRCexplorer2 [37], CIRI2 [56] and Hyb [45]. CIRCexplorer2 and CIRI2 are two of the most widely utilized pipelines for circRNA identification and found approximately 82,000 and 180,000 distinct circRNAs across these tissues, respectively (Figure 1b). Over 72,000 circRNAs ( $88 \%$ of CIRCexplorer 2 circRNAs and $40 \%$ of CIRI2 circRNAs) were found in common between these pipelines, with CIRI2 identifying a larger number of very lowly abundant circRNAs (1 or 2 backsplice junction counts) accounting for the almost 100,000 extra unique circRNAs (Figure S1d). Hyb, a pipeline designed to identify chimeric RNA reads and which was used to identify circRNAs in a previous report [45], was employed here to identify circRNAs genome-wide. In this study, Hyb identified over 73,000 circRNAs; approximately $45 \%$ of these were also identified in either CIRCexplorer 2 or CIRI2. These trends are consistent with previous reports showing variability across pipelines for predicting circRNAs [44]. Further analyses were focused on the subset of 13,209 high-confidence circRNAs meeting strict thresholds, including normalized expression minimum (CMP > 0.01); a minimum of two unique reads in at least three samples and those commonly identified in all three pipelines. 
(a)

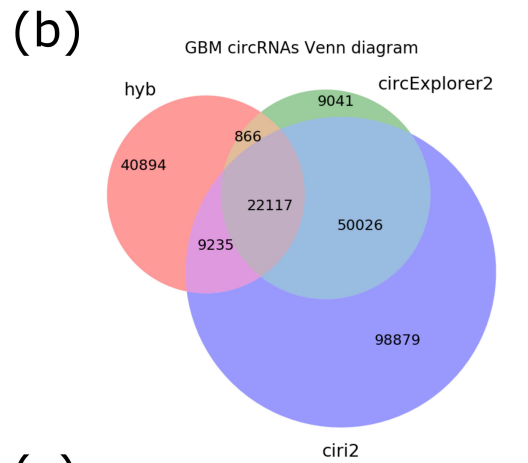

(c)

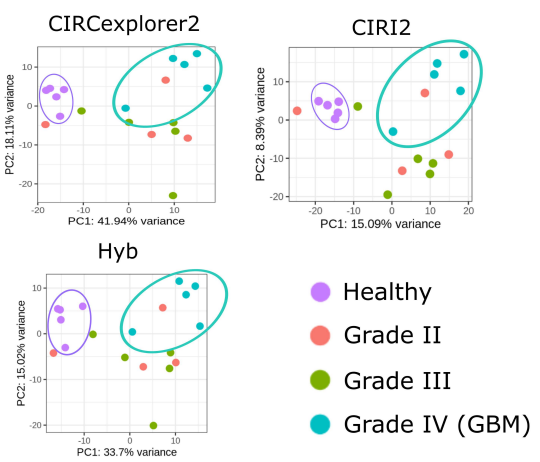

(d)

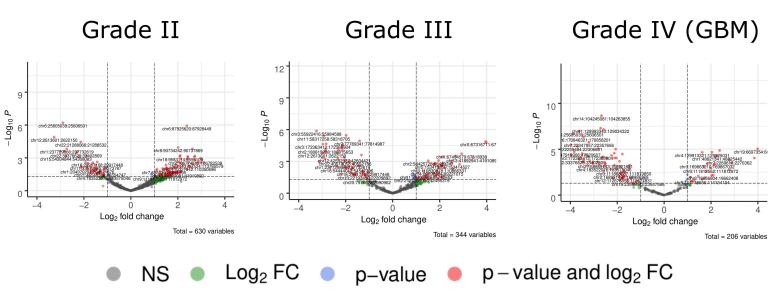

(e)

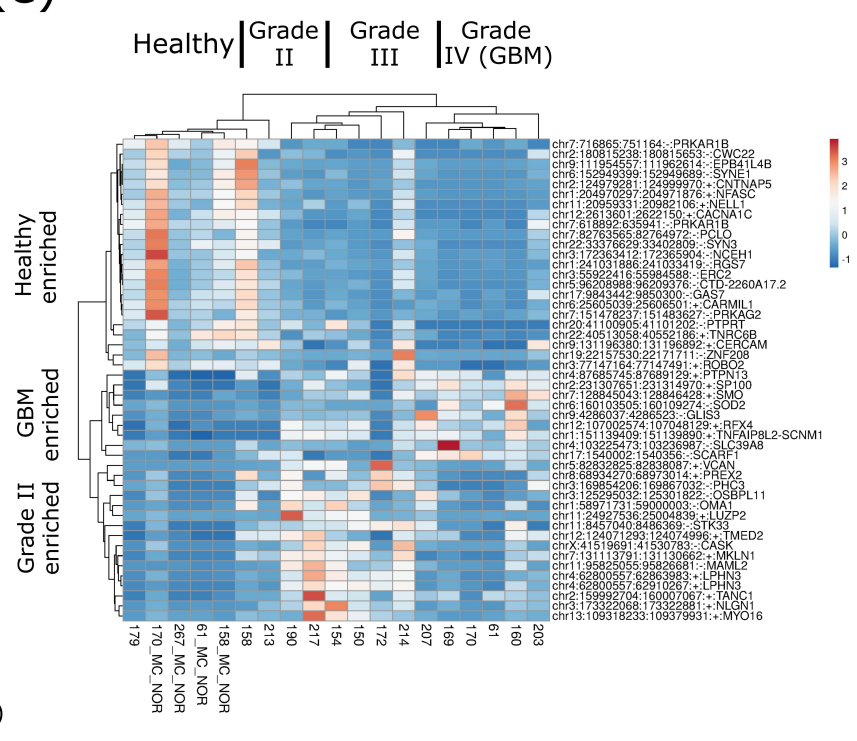

Figure 1. Circular RNA (circRNA) profiling of human gliomas can stratify glioblastoma (GBM) tumors. (a) Canonical linear splicing versus non-canonical, back-splicing producing mRNA and circRNA, respectively. Arc highlights the location of the backsplice junction. (b) Scaled Venn diagram showing total number of circRNAs identified from all 19 brain, circRNA-seq libraries using three different circRNA identification pipelines, CIRCexplorer2, CIRI, and Hyb. (c) Two-dimensional PCA plot utilizing circRNA-seq output from CIRCexplorer2 on 19 samples across healthy, grade II, III, and IV (GBM) gliomas. Ellipses included to highlight stratification of healthy tissue (purple) and grade IV tumor (blue) for each of the three circRNA prediction pipelines. (d) Expanded volcano plot showing circRNAs regulated between healthy tissue and grade II (left panel), grade III (middle panel) and grade IV, GBM (right panel). (e) Heat map showing representative examples of circRNAs specific to healthy brain tissue, grade-specific (grade II and grade IV (GBM)) using CIRCexplorer2. The color scale bar represents the log-transformed normalized reads.

Interestingly, despite the distinct population of circRNA identified between the three pipelines, the PCA plots for the unfiltered lists are similar and each showed a clear separation between healthy and grade IV (GBM) samples (Figure 1c). However, grade II and grade III tumors did not show a clear separation in any of the analyses. This ability to stratify GBM tumor from normal tissue based on circRNA profile alone suggests that some circRNAs may constitute bona fide biomarkers of GBM. From our high-confidence population of circRNAs averaged within each sample type, we identified circRNA candidates which are altered in expression compared with healthy tissue, for grade II, grade III, and grade IV (GBM) glioma tumors (Figure 1d). There are more circRNAs which are significantly downregulated in GBM compared to healthy tissues. Heat map representation clearly shows that, while some specific circRNA candidates are enriched in healthy tissue or distinct glioma grades, there is biological variability between patients (Figure 1e; Table S2). One example, circGLIS3, was also previously shown to be more highly expressed in GBM compared to normal tissue [57]. While beyond the scope of this study, our study supports the finding that these, and other, circRNAs could represent not only diagnostic, but prognostic biomarkers of gliomas and GBM. 


\subsection{Microexons Are Identified in Circrna Sequencing}

While yet to be reported for circRNAs, incorporation of a microexon (ME) - defined herein as an exon comprising 3-30 nt in length-into circRNAs may occur within the body of the circRNA or at the backsplice junction (Figure 2a). Therefore, to see if circRNAs could incorporate MEs, we utilized a reference database of 1239 known MEs localized to specific genomic coordinates within mRNAs from Irimia et al. [15] and searched for overlap with circRNAs from our glioma RNA-seq data. To maximize the accuracy of ME-circRNA prediction, we only included those known MEs whose expression was found in our circRNA-seq data with reads crossing the ME and both flanking exons from the same gene. An example of this is the known ME in SUPT5H which is incorporated into reads spanning canonical exons 4-5 (Figure S1a).

(a)

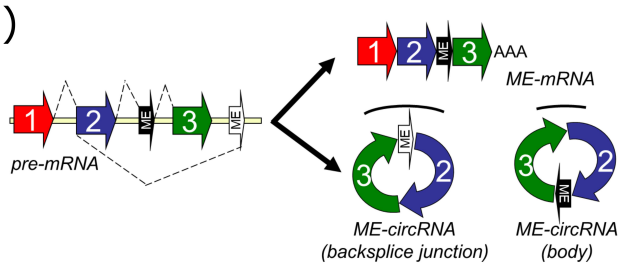

(c)

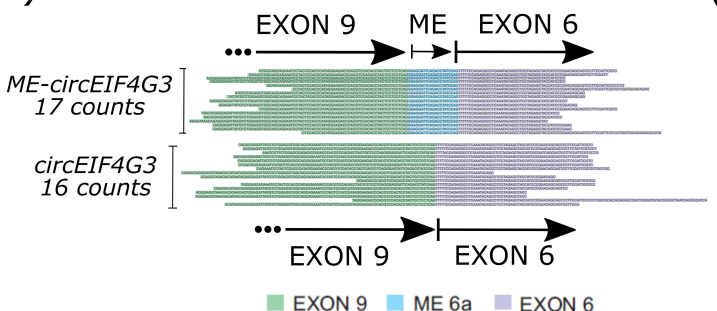

(e)

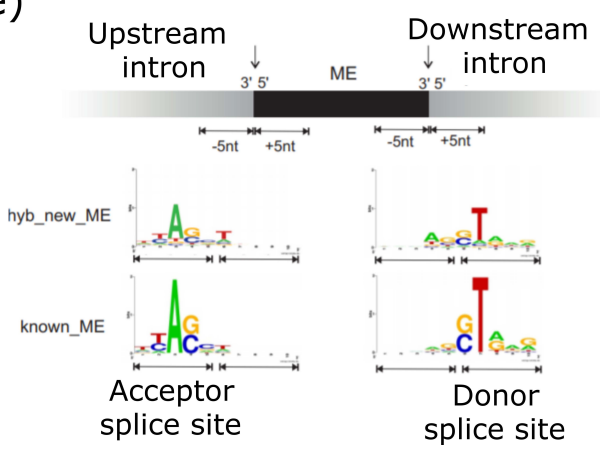

(b) Known MEs overlapping circRNAs

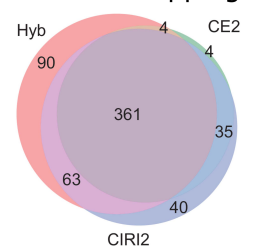

(d)

Proportion of ME-circRNAs with matched cognate circRNAs

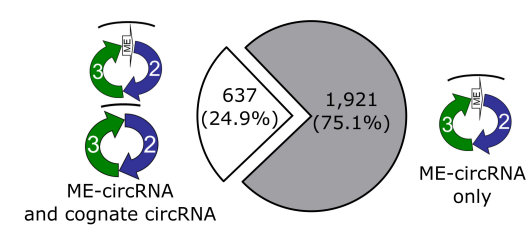

(f)

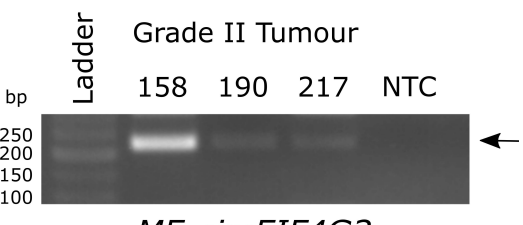

ME-cirCEIF4G3

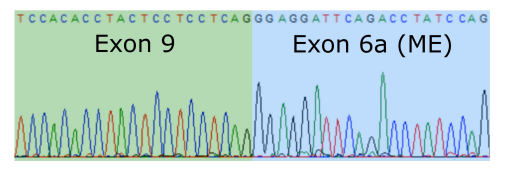

Figure 2. Microexons are included in circRNAs. (a) Incorporation of microexons (ME), which are defined here as 3-30 nt sequences into mRNA and circRNA. Note that MEs in circRNAs can fall into the backsplice junction (unfilled exon) or into the body (filled exon) of the circRNA. (b) circRNAs overlapping known microexon sequences for three pipelines. (c) Trimmed reads mapped to EIF4G3 which cover the circRNA backsplice junction across exon 9 and exon 6 (circEIF4G3, 16 counts) and the same junction with a 21nt ME (ME-circEIF4G3, 17 counts) from healthy brain tissue from patient \#170. (d) De novo identification of ME-circRNAs using Hyb. Pie chart showing proportion of ME-circRNAs with matched cognate circRNA (637/2558; 24.9\%) and orphan ME-circRNAs (1921/2558; 75.1\%). (e) Sequence motif enrichment using WebLogo (ver2.8.2) around splice sites of predicted (upper) and known (lower) MEs in ME-circRNAs. The lines under each graph indicates the $5 \mathrm{nt}$ of the intron side and the exon side of the ME splice site with the upstream acceptor splice site on the left and the downstream donor splice site on the right. (f) Upper, Agarose gel showing RT-PCR amplification of ME-circEIF4G3 in RNA from tumor samples \#158, \#190 and \#217 and absence in non-template control (NTC). Lower, Sanger sequencing of PCR product across backsplice junction between the ME (blue shading, exon 6a) and exon 9 (green shading). 
After filtering for expression of the ME, we identified 696 of these MEs (or 56\%) overlapping our high-confidence circRNAs with, on average, $8.5 \%$ of these located at either the $5^{\prime}$ or $3^{\prime}$ exon comprising the circRNA backsplice junction and the remainder within the body of the circRNA (Table S3). As depletion of linear RNA is accomplished by pre-digestion of the RNA with RNase R prior to library preparation (Figure S1b), this suggests that these MEs are not derived from mRNA sequences, but most likely exist within the enriched circRNAs themselves. In support of this, the gold-standard ME in the REST mRNA could not be detected following RNase $\mathrm{R}$ digestion in any of the healthy or tumor samples, or by RT-PCR (Figure S1b), while circLRBA was detectable. Surprisingly, despite ME-circRNAs not being reported to date, all three pipelines identified 361 common ME-circRNAs, covering $29 \%$ of known MEs from Irimia et al. [15]. The circRNAs predicted from the Hyb pipeline overlapped with the greatest number of known MEs (518), followed by CIRI (499) and CIRCexplorer2 (404) (Figure 2b).

This is the first report, to our knowledge, of ME-cirRNAs, with CIRCexplorer2, CIRI2, and Hyb identifying previously annotated MEs in the reference genome, rather than new ME-circRNAs. As this indicates there is greater complexity within the cellular repertoire of circRNAs than previously appreciated, we expanded our search to identify de novo MEs in this circRNA-seq data.

\subsection{Using Hyb Identifies Novel MEs and ME-circRNAs}

The Hyb pipeline was originally designed to identify de novo RNA chimeras in an unbiased manner from RNA-seq [45], and has been used to verify 190 of the 239 circRNAs from Memczak et al. [58]. Therefore, we employed Hyb to identify MEs at the backsplice junction of circRNAs. In accordance with previous characterizations, MEs were specified to comprise sequences of 3-30 nt in length between exons which form the circRNA backsplice junction. Across these libraries, Hyb predicted a total of 2,558 ME-circRNAs with the ME at the backsplice junction (Table S4), which is approximately $3.5 \%$ of all circRNAs identified using the Hyb pipeline. In a similar result, of the 518 known MEs from Irimia et al. [15] shown to overlap circRNAs using Hyb (Figure 2b), 30 of these were shown to exist at the backsplice junction (Table S3), or 5.8\% of known ME-circRNAs.

As the first report of MEs in circRNAs, we performed two in silico analyses to assess the predictive accuracy of the Hyb pipeline. Taking all reads supporting ME-circRNAs using the Hyb pipeline across the 19 brain tissue samples (11,888 reads), we isolated the ME sequence. These were then either (1) randomly shuffled into the backsplice junction of one of the circRNAs (without rearrangement of the ME sequence) or (2) the ME sequences ( $>6 \mathrm{nt}$ in length) was scrambled and reinserted into the same circRNA. These tests were to mimic an artefactual or random inclusion of a sequence which could arise by chance or library preparation artefact and give rise to false positive ME-circRNAs. We then aligned these shuffled or scrambled ME-circRNAs with the original ME-circRNA reads from the Hyb output. If these shuffled or scrambled ME-circRNA reads were still detected at high levels, then our Hyb pipeline could not be considered highly accurate due to a high false positive rate. On the contrary, only 172 shuffled ME-circRNA reads (1.44\%) and 38 scrambled ME-circRNA reads $(0.3 \%)$ could be found by chance in our ME-circRNA database. This suggests a predictive accuracy exceeding $98.5 \%$, providing confidence in the Hyb pipeline for identifying bona fide ME-circRNAs.

One example of a novel ME-circRNA is evident from the trimmed reads from the EIF4G3 gene (Figure 2c). For this candidate, both the canonical circRNA which has a backsplice junction comprising exon 9 and exon 6 (circEIF4G3) and the equivalent ME-circRNA (ME-circEIF4G3) incorporating a 21nt ME (called exon 6a) could be detected with 16 and 17 reads, respectively, in the healthy brain tissue of GBM patient \#170 and 3 and 0 reads in the matched GBM tumor. This provides evidence that Hyb is the only pipeline currently with the capacity to capture this non-trivial panel of potentially functional ME-circRNA candidates.

For the de novo ME-circRNAs identified by Hyb, we wanted to see how many cognate circRNAs were present which shared the same backsplice junction as a ME-circRNA, but lacking the ME. We found 629 examples (24.9\% of ME-circRNAs) where both the circRNA and the ME-circRNA were 
present (Figure 2d). In addition to ME-circEIF4G3 (Figure 2c), another example of this was circRIMS1 which had a canonical circRNA comprising exons 27-29 (chr6: 73016960-73043538) and ME-circRIMS1 which also incorporates a 30nt ME at the backsplice junction between exons27-29. However, for the remaining $75 \%$ of ME-circRNAs, these did not have a cognate circRNA, suggesting a large proportion of mutually-exclusive circRNA splicing events with respect to MEs.

We sought to identify the most likely genomic coordinates for the novel ME sequences predicted by Hyb in the 2558 ME-circRNAs. Due to small ME sequences mapping at multiple loci, we focused only on MEs $6 \mathrm{nt}$ or longer and wanted to establish whether canonical intron splicing motifs flanked these MEs. For these MEs, the strongest hit was identified by prioritizing following three criteria: matching sequence must be on the same strand as the circRNA, within the same gene and in closest proximity to the $5^{\prime}$ or $3^{\prime}$ exon comprising the canonical circRNA backsplice junction. Once this list of putative coordinates was generated, we performed sequence enrichment motif analysis using WebLogo (ver2.8.2) to assess whether the canonical GT-AG rule for spliced introns is abided by for MEs [59]. By looking at the $5 \mathrm{nt}$ either side of both the start and end of the ME, we identified that the dinucleotides at the first two intron positions (donor splice site) and last two intron positions (acceptor splice site) flanking the putative ME were enriched for GT and AG, respectively, providing confidence in our predicted genomic coordinates for these spliced MEs (Figure 2e).

Validation of one putative ME-circRNA identified by the Hyb pipeline-ME-circEIF4G3 comprising exons 6a-9, with the ME at the $5^{\prime}$ end of the backsplice junction-was undertaken by RT-PCR. RNase R-treated RNA from grade II tumors underwent reverse transcription and RT-PCR using an oligonucleotide anchored in the $21 \mathrm{nt} \mathrm{ME}$ itself (exon 6) and another divergent oligonucleotide in exon 10. The $218 \mathrm{bp}$ PCR product corresponding to the ME-circRNA was detected in the tumors and at the same relative abundance of that ME from within each RNA-seq dataset (\#158 had 307 ME counts, \#190 had 114 and \#217 had 130 ME counts), with Sanger sequencing confirming the ME comprises the backsplice junction (Figure 2f). As further evidence for the existence of ME-circRNAs identified by Hyb, we also performed RT-PCR and Sanger sequencing for ME-circDPY19L1 (Figure S2). Using oligonucleotides anchored in exons 6 and 3 flanking the ME, ME-circDPY19L1, unlike $M E$-circEIF4G3, did not exhibit a matched cognate circRNA as there was a single amplicon of the anticipated size when amplified from two different tumor samples.

\subsection{Decrease in ME-circRNAs in GBM Is Correlated with SRRM4 Expression}

Comparing matched normal and GBM datasets we, like others, observed a decrease in the total number of circRNA backsplice junction counts across all three pipelines. Specifically, we identified 32,617 circRNAs in healthy tissues, while only 28,842 circRNAs were detected in GBM tissues (11\% decrease). This was similar when looking at the high-confidence subset of 13,209 circRNAs with 11,773 found in healthy tissue and 10,621 in grade IV tumors (9.8\% decrease; Figure S3). When focusing on MEs alone and ME-circRNAs identified by Hyb, there was a 22\% and 37\% decrease between the healthy and grade IV tumors, respectively. Therefore, we sought to identify which protein factors known to regulate ME inclusion in the brain could be correlated with this greater decrease in ME-circRNAs.

Probing the GEPIA interactive web server, which incorporates over 18,000 matched tumor and normal samples from the TCGA and GTEx projects [60], we identified SRRM4 was significantly reduced in both GBM tumors and low-grade gliomas compared with healthy tissues (Figure S4a). We also checked the expression of U2AF2 and RBFOX1, two other known factors implicated in neuronal ME splicing [10], by semi-quantitative PCR between the three tumor grades. This showed no clear visible alteration in expression across the grades for RBFOX1 or U2AF2, but SRRM4 exhibited a noticeable decrease (Figure S4b). Therefore, we performed qRT-PCR on the five healthy and five GBM tissues which we previously sequenced (Table 1) to quantify the expression levels of the known neuronal splicing factor SRRM4 and its family members SRRM3 (neuronal-specific) and the ubiquitously-expressed SRRM1 and SRRM2. Comparing expression by qRT-PCR, SRRM4 was the only family member to be significantly decreased (One-way ANOVA, Mann-Whitney test, $p=0.023$ ) 
in expression in GBM compared with the normal brain tissue (Figure 3a). This lower expression of $S R R M 4$ in the core tumor compared with healthy tissue correlates well with the expression profiling from the publicly available IVYGap database.

(a)

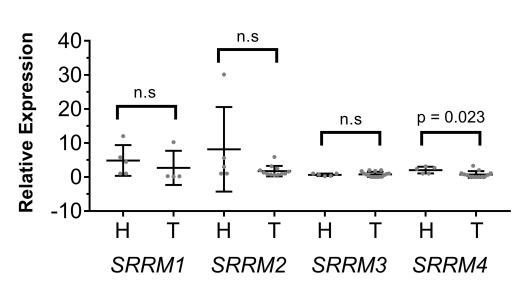

(d)

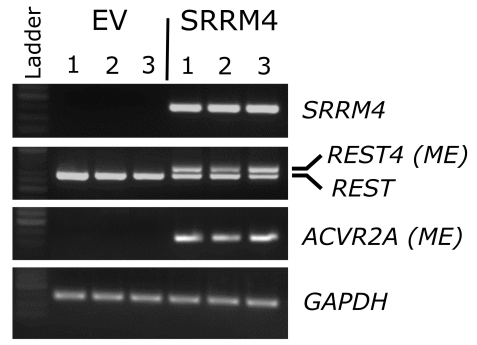

(b)

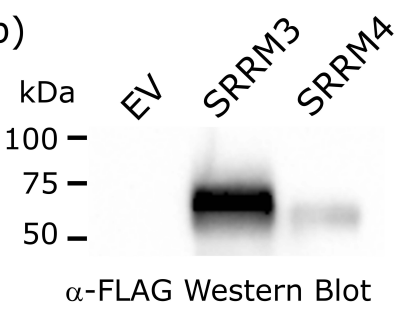

(e)

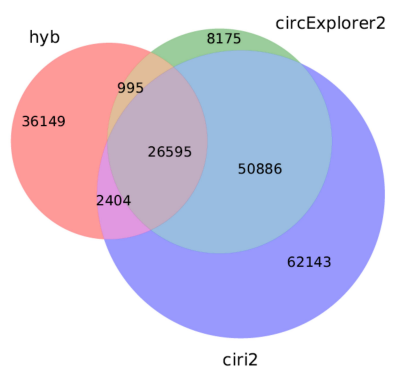

(c)

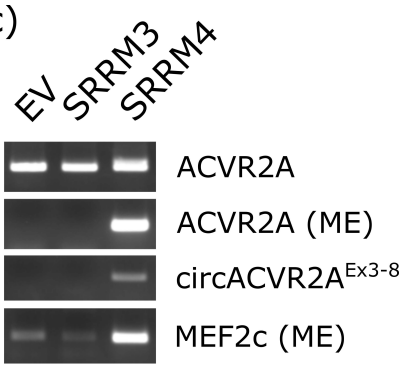

(f)

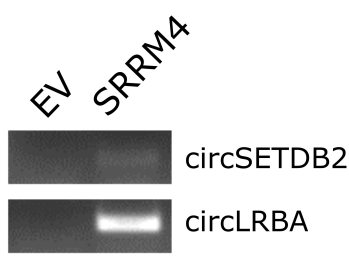

(g)

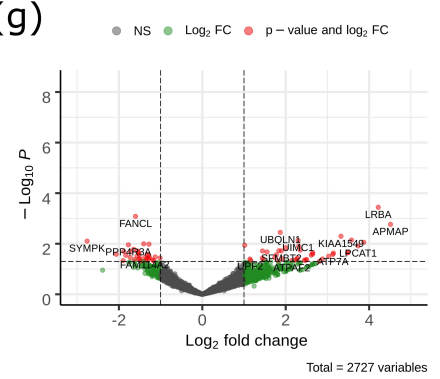

(h)

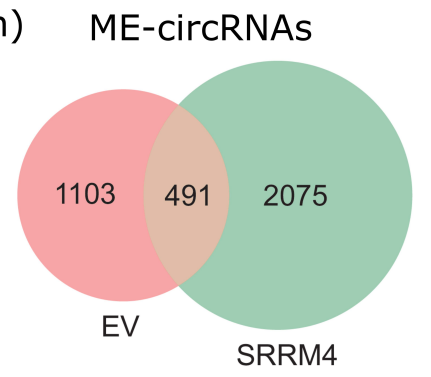

(i)

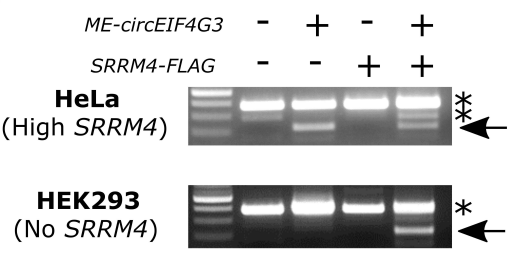

Figure 3. Serine/arginine repetitive matrix 4 (SRRM4) is a bona fide circRNA and ME-circRNA biogenesis factor. (a) qRT-PCR comparing relative expression of SRRM transcripts between healthy brain tissue $(\mathrm{H})$ and GBM tumor tissue (T). Normalized to GAPDH, $\mathrm{n}=4-5$ tissue samples. One-way ANOVA, Mann-Whitney test was performed to assess significance. n.s.: no significant difference $(p>0.05)(\mathbf{b})$ Western blot using FLAG antibody on cell protein lysates from HEK293 cells transiently transfected with pcDNA3.1 (EV), pcDNA3.1::SRRM3-FLAG (SRRM3) or pcDNA3.1::SRRM4-FLAG (SRRM4). Bands of expected size for SRRM3 (70 kDa) and SRRM4 (65 kDa). (c) RT-PCR for ACVR2A linear (ACVR2A), and SRRM4-specific ACVR2A RNA with ME, circACVR2A (comprising exons 3-8) and increase in MEF2C linear RNA with ME. (d) RT-PCR on 3 biological replicates of HEK293 cells with EV, or SRRM4 overexpression for SRRM4, REST (2 bands, with upper band representing the inclusion of ME in REST4), ACVR2A (ME) and GAPDH used as loading control. (e) Scaled Venn diagram showing total number of circRNAs identified across the three replicate EV and SRRM4 overexpression circRNA-seq libraries using three different circRNA identification pipelines, CIRCexplorer2, CIRI and Hyb. (f) RT-PCR for two novel SRRM4-driven circRNAs showing absence in HEK293 EV cells. (g) Volcano plot comparing circRNA expression between EV and SRRM4 overexpression. (h) Scaled Venn diagram showing ME-circRNAs, with the ME present at the backsplice junction, identified using Hyb. (i) RT-PCR showing the dependence on SRRM4 for expression of ME-circEIF4G3 from transgenic circRNA overexpression construct. Top, HeLa cells (with high levels of SRRM4 expression) could produce ME-circEIF4G3 with and without SRRM4 overexpression $24 \mathrm{~h}$ post-transfection. Bottom, HEK293 cells (lacking SRRM4 expression) could only produce the circRNA if SRRM4 was co-transfected. * are non-specific RT-PCR products. 


\subsection{SRRM4 Is a Bona Fide circRNA and ME-circRNA Biogenesis Factor}

To investigate whether SRRM4 could regulate ME inclusion in circular RNAs, we overexpressed SRRM4 in HEK293 cells as per Torres-Mendez et al. [61] and Nakano et al. [62]. These cells were chosen as they lack SRRM4 expression, allowing us to delineate the consequence of this gene on circular RNA identity. A C-terminal FLAG-tagged construct was made for SRRM4 and SRRM3 (as a control) and transfected into HEK293 cells. This produced a protein of the anticipated size for each construct by western blotting with anti-FLAG antisera (Figure $3 b$ ). RT-PCR was performed on matched RNA samples to detect $A C V R 2 A$ mRNA, ME-ACVR2A and $\operatorname{circ} A C V R 2 A$. It was found that while there was not a significant difference in baseline expression of $A C V R 2 A$ mRNA between EV, SRRM3 and SRRM4 overexpressing HEK293 cells, we noted a novel ME-containing amplicon using oligonucleotides that flank the ME (Figure 3c, upper panel) producing the wild-type product (196 bp) in all lines, but a larger, ME-containing band (220 bp) uniquely in the SRRM4 overexpressor. This was confirmed using an oligonucleotide anchored in the ME itself, with the product only seen in the SRRM4 overexpressor line, as expected (Figure 3c). Furthermore, a unique circRNA from $A C V R 2 A$, comprising exons 3-8, was found in the SRRM4 overexpressor alone (Figure 3c).

RT-PCR on three biological replicates showed the presence of SRRM4 only in the overexpression lines, not the EV cell lines (Figure 3d). Furthermore, the inclusion of the microexon in the REST transcript driven by SRRM4 was investigated by RT-PCR as per Nakano et al. [63]. These primers flank exon 4 of REST and produce a $225 \mathrm{bp}$ product from the active REST mRNA and a $309 \mathrm{bp}$ product from the REST4 isoform. In the presence of the empty vector, only the shorter amplicon was detected, while all three biological replicates of SRRM4-FLAG overexpression saw the REST4 amplicon also detected.

RNA was harvested from HEK293 cells, 48hrs post-transfection with either pcDNA3.1 (empty vector, EV) or pcDNA3.1::SRRM4. CircRNA-seq was performed as for GBM cells on three biological replicates of EV and SRRM4 overexpression and the resultant libraries sequenced on the Illumina NextSeq550. The distribution profile of circRNAs predicted with the three pipelines CIRCexplorer2, CIRI2 and Hyb (Figure 3e) were similar in distribution, but fewer in total numbers, compared with the glioma circRNA-seq (Figure 2b). CircRNAs predicted by Hyb, showed a 10\% increase in the count of unique circRNAs identified from 43,380 circRNAs in EV to 47,869 circRNAs with SRRM4 overexpression. Two novel, SRRM4-driven circRNAs-circLRBA and circSETDB2-were validated by RT-PCR as being absent in HEK293 cells and present in the SRRM4 overexpressing cells (Figure 3f). Furthermore, an enhanced Volcano Plot of this data highlights more circRNAs are quantitatively increased in expression along with SRRM4 in HEK293 cells, rather than decreased or lost (Figure 3g). However, a more significant increase was found in ME-circRNAs (61\% increase) with SRRM4 overexpression, including over 2000 unique ME-circRNAs at the backsplice junction (Figure 3h; Table S5). This agrees with SRRM4 being a master regulator of ME inclusion in mRNAs and that it recapitulates these effects with ME-circRNAs. One example of this was a microexon in the ACVR2A gene which could be amplified by RT-PCR uniquely in all three SRRM4 overexpression replicates and not in the empty vector (Figure 3c,d). While MEF2C mRNA containing a ME was present in EV and SRRM3 lines, it visibly increased in abundance with SRRM4 expression, suggesting SRRM4 can boost ME inclusion when there is a basal level of expression (Figure 3c).

While this evidence shows a number of circRNAs and ME-circRNAs can be regulated by SRRM4 expression, we wanted to validate the dependency of a ME-circRNA on SRRM4 expression. To address this, we focused on a ME-circRNA from our GBM data (Figure 2e), ME-circEIF4G3, but notably absent from the HEK293 data. We constructed a circRNA overexpression vector to express ME-circEIF4G3, which was transiently transfected into HeLa cells, a cell line known for possessing one of the highest expression levels of SRRM4 expression from the Human Proteome Atlas [64] (Figure S5a), but did not produce this ME-circRNA de novo (Figure 3i). This is not unexpected, given the cell-type specific nature of circRNAs [65]. An RT-PCR product specific for this circRNA was detected (and confirmed by Sanger sequencing) only upon expression of the ME-circEIF4G3 transgenic construct (Figure 3i). 
Co-transfection of the circRNA construct with pcDNA3.1::SRRM4 in this line did not significantly increase the level of this ME-circRNA. This experiment was repeated in HEK293 cells, which we show lacks expression of SRRM4 (Figure 3c, Figure S5a). Remarkably, despite the use of perfectly reverse complementary introns of $800 \mathrm{bp}$ in length in the overexpression construct, ME-circEIF4G3 was only produced upon co-transfection with pcDNA3.1::SRRM4 in HEK293 cells (Figure 3i). This provides strong evidence for the role of SRRM4 as a bona fide circRNA and ME-circRNA biogenesis factor, suggesting it is necessary in both HeLa and HEK293 cells, and sufficient in HEK293 cells for biogenesis of ME-circEIF4G3.

\section{Discussion}

To our knowledge, this is the first report of microexons (MEs) detected in circular RNAs. We identified thousands of circRNAs containing MEs, which we deem ME-circRNAs, which were previously unidentified, or unappreciated. These should now be recognized as a non-trivial sub-family of non-coding RNAs and be a focus of new research. In the most comprehensive study to date on diverse circular RNA composition, Zhang et al. [37] identified novel exons being incorporated into circRNAs but did not report MEs as a non-canonical component.

MEs are known to play functional roles in development, with emerging evidence of their association with diseases including autoimmune conditions and cancer [14]. In particular, SRRM4, implicated here as a bona fide circRNA and ME-circRNA biogenesis factor, was shown to regulate a splicing program critical in the trans-differentiation and progression of neuroendocrine prostate cancer $[38,39]$. MEs within the EIF4G1 and EIF4G3 transcripts were shown to be misregulated in autism patients and control synaptic translation and cognition contributing to pathogenesis of this condition [66]. Despite the focus of these reports being on the alternative splicing of the coding transcriptome, our study opens the possibility that ME-circRNAs, like other circRNAs, could play a pathogenic role in cancer. With respect to glioma, we identified a significant correlation $(p<0.0001)$ between low SRRM4 expression and poor survival in GBM and low-grade glioma across the Chinese Glioma Genome Atlas (CGGA), The Cancer Genome Atlas (TCGA) and Repository of Molecular Brain Neoplasia Data (Rembrandt) datasets (Figure S5b-d). We show here that ME-circEIF4G3 is controlled by SRRM4 and, given the down-regulation of SRRM4 in gliomas and association with poor survival outcomes, this may warrant further investigation of the role of this and other ME-circRNAs in gliomagenesis.

The expression of SRRM4 in HEK293 cells, which do not express this neural-specific transcript, was a deliberately controlled experiment and with that we found thousands of circRNAs and ME-circRNAs were altered in expression. Interestingly, many of these were intermediate-lowly expressed circRNAs with the four most abundant circRNAs unchanged (circSPECC1 (Figure S1a), circMAN1A2, circSMARCA5, and circHIPK3). This is also the first report showing a dependence of a transgenic circRNA overexpression construct encoding circME-EIF4G3, requiring co-expression of SRRM4 (or in a cell line, like HeLa cells, with high baseline expression of SRRM4) to generate the circRNA product. This may be a consequence of the ME perturbing the secondary structure of the RNA, despite the presence of reverse complementary introns which are normally sufficient to drive circRNA expression. Further research will need to be undertaken on the newly identified ME-circRNAs including their unique regulation, impact on RNA secondary structure and function.

Supplementary Materials: The following are available online at http://www.mdpi.com/2073-4409/9/11/2488/s1, Figures S1-S5. Table S1: Oligonucleotides used in this study. Table S2: Normalized expression levels of circRNAs represented by heatmap. Table S3: Known ME overlap with circRNAs predicted from three pipelines. Table S4: CircRNAs overlapping de novo MEs found using Hyb pipeline in glioma and healthy brain tissue. Table S5: CircRNAs overlapping de novo MEs found using Hyb pipeline in HEK293 cells with SRRM4 overexpression.

Author Contributions: Conceptualization, V.M.C. and S.J.C.; methodology, V.M.C., M.G., S.M., R.J.O., T.P., and S.J.C.; software, M.G., S.M., and T.P.; formal analysis, V.M.C., M.G., S.M., B.W.S., T.P., S.M., and S.J.C.; resources (clinical information and patient sample curation), R.J.O., S.P., and G.K.; investigation, V.M.C., B.W.S., M.G., T.P., and S.J.C.; data curation, S.M. and R.J.O.; writing—original draft preparation, S.J.C.; writing-review 
and editing, V.M.C., M.G., B.W.S., R.J.O., T.P., S.M., S.P., G.K., and S.J.C.; project administration, S.J.C.; funding acquisition, S.J.C., V.M.C., R.J.O; supervision, S.J.C.; visualization, V.M.C., M.G., S.M., B.W.S., T.P., and S.J.C.; validation, V.M.C., M.G., S.M., B.W.S., T.P., and S.J.C. All authors have read and agreed to the published version of the manuscript.

Funding: This research and APC was funded by National Health and Medical Research Council, GNT1144250 to S.J.C.; Salary for S.J.C. was provided from the Australian Research Council Future Fellowship Scheme, FT160100318; Flinders Foundation Health Seed Grant to S.J.C., V.M.C., and G.K.

Acknowledgments: We would like to acknowledge the unsung champions of our research, the glioma patients who donate their brain material. We hope this accelerates the search for a cure.

Conflicts of Interest: The authors declare no conflict of interest.

\section{References}

1. Cvitkovic, I.; Jurica, M.S. Spliceosome Database: A tool for tracking components of the spliceosome. Nucleic Acids Res. 2013, 41, D132-D141. [CrossRef] [PubMed]

2. Chen, W.; Moore, M.J. Spliceosomes. Curr. Biol. 2015, 25, R181-R183. [CrossRef] [PubMed]

3. Sibley, C.R.; Blazquez, L.; Ule, J. Lessons from non-canonical splicing. Nat. Rev. Genet. 2016, 17, 407-421. [CrossRef] [PubMed]

4. Wang, E.T.; Sandberg, R.; Luo, S.; Khrebtukova, I.; Zhang, L.; Mayr, C.; Kingsmore, S.F.; Schroth, G.P.; Burge, C.B. Alternative isoform regulation in human tissue transcriptomes. Nature 2008, 456, 470-476. [CrossRef]

5. Chen, K.; Dai, X.; Wu, J. Alternative splicing: An important mechanism in stem cell biology. World J. Stem Cells 2015, 7, 1-10. [CrossRef]

6. Yeo, G.; Holste, D.; Kreiman, G.; Burge, C.B. Variation in alternative splicing across human tissues. Genome Biol. 2004, 5, R74. [CrossRef]

7. Scotti, M.M.; Swanson, M.S. RNA mis-splicing in disease. Nat. Rev. Genet. 2016, 17, 19-32. [CrossRef]

8. Barash, Y.; Calarco, J.A.; Gao, W.; Pan, Q.; Wang, X.; Shai, O.; Blencowe, B.J.; Frey, B.J. Deciphering the splicing code. Nature 2010, 465, 53-59. [CrossRef]

9. Coelho, M.B.; Smith, C.W.J. Regulation of alternative pre-mRNA splicing. Methods Mol. Biol. Clifton N. J. 2014, 1126, 55-82. [CrossRef]

10. Ustianenko, D.; Weyn-Vanhentenryck, S.M.; Zhang, C. Microexons: Discovery, regulation, and function. Wiley Interdiscip. Rev. RNA 2017, 8. [CrossRef]

11. Volfovsky, N.; Haas, B.J.; Salzberg, S.L. Computational discovery of internal micro-exons. Genome Res. 2003, 13, 1216-1221. [CrossRef] [PubMed]

12. Yan, Q.; Weyn-Vanhentenryck, S.M.; Wu, J.; Sloan, S.A.; Zhang, Y.; Chen, K.; Wu, J.Q.; Barres, B.A.; Zhang, C. Systematic discovery of regulated and conserved alternative exons in the mammalian brain reveals NMD modulating chromatin regulators. Proc. Natl. Acad. Sci. USA 2015, 112, 3445-3450. [CrossRef] [PubMed]

13. Li, Y.I.; Sanchez-Pulido, L.; Haerty, W.; Ponting, C.P. RBFOX and PTBP1 proteins regulate the alternative splicing of micro-exons in human brain transcripts. Genome Res. 2015, 25, 1-13. [CrossRef] [PubMed]

14. Gonatopoulos-Pournatzis, T.; Blencowe, B.J. Microexons: At the nexus of nervous system development, behaviour and autism spectrum disorder. Curr. Opin. Genet. Dev. 2020, 65, 22-33. [CrossRef]

15. Irimia, M.; Weatheritt, R.J.; Ellis, J.; Parikshak, N.N.; Gonatopoulos-Pournatzis, T.; Babor, M.; Quesnel-Vallières, M.; Tapial, J.; Raj, B.; O’Hanlon, D.; et al. A highly conserved program of neuronal microexons is misregulated in autistic brains. Cell 2014, 159, 1511-1523. [CrossRef]

16. Raj, B.; Irimia, M.; Braunschweig, U.; Sterne-Weiler, T.; O’Hanlon, D.; Lin, Z.-Y.; Chen, G.I.; Easton, L.E.; Ule, J.; Gingras, A.-C.; et al. A global regulatory mechanism for activating an exon network required for neurogenesis. Mol. Cell 2014, 56, 90-103. [CrossRef]

17. Calarco, J.A.; Zhen, M.; Blencowe, B.J. Networking in a global world: Establishing functional connections between neural splicing regulators and their target transcripts. RNA 2011, 17, 775-791. [CrossRef]

18. Raj, B.; O’Hanlon, D.; Vessey, J.P.; Pan, Q.; Ray, D.; Buckley, N.J.; Miller, F.D.; Blencowe, B.J. Cross-Regulation between an Alternative Splicing Activator and a Transcription Repressor Controls Neurogenesis. Mol. Cell 2011, 43, 843-850. [CrossRef]

19. Magin, A.; Lietz, M.; Cibelli, G.; Thiel, G. RE-1 silencing transcription factor-4 (REST4) is neither a transcriptional repressor nor a de-repressor. Neurochem. Int. 2002, 40, 195-202. [CrossRef] 
20. Vuong, C.K.; Black, D.L.; Zheng, S. The neurogenetics of alternative splicing. Nat. Rev. Neurosci. 2016, 17, 265-281. [CrossRef]

21. Sanger, H.L.; Klotz, G.; Riesner, D.; Gross, H.J.; Kleinschmidt, A.K. Viroids are single-stranded covalently closed circular RNA molecules existing as highly base-paired rod-like structures. Proc. Natl. Acad. Sci. USA 1976, 73, 3852-3856. [CrossRef] [PubMed]

22. Nigro, J.M.; Cho, K.R.; Fearon, E.R.; Kern, S.E.; Ruppert, J.M.; Oliner, J.D.; Kinzler, K.W.; Vogelstein, B. Scrambled exons. Cell 1991, 64, 607-613. [CrossRef]

23. Kristensen, L.S.; Andersen, M.S.; Stagsted, L.V.W.; Ebbesen, K.K.; Hansen, T.B.; Kjems, J. The biogenesis, biology and characterization of circular RNAs. Nat. Rev. Genet. 2019, 20, 675-691. [CrossRef] [PubMed]

24. Jeck, W.R.; Sorrentino, J.A.; Wang, K.; Slevin, M.K.; Burd, C.E.; Liu, J.; Marzluff, W.F.; Sharpless, N.E. Circular RNAs are abundant, conserved, and associated with ALU repeats. RNA 2013, 19, 141-157. [CrossRef]

25. Ashwal-Fluss, R.; Meyer, M.; Pamudurti, N.R.; Ivanov, A.; Bartok, O.; Hanan, M.; Evantal, N.; Memczak, S.; Rajewsky, N.; Kadener, S. circRNA biogenesis competes with pre-mRNA splicing. Mol. Cell 2014, 56, 55-66. [CrossRef]

26. Conn, S.; Pillman, K.; Toubia, J.; Conn, V.; Salmanidis, M.; Phillips, C.; Roslan, S.; Schreiber, A.; Gregory, P.; Goodall, G. The RNA Binding Protein Quaking Regulates Formation of circRNAs. Cell 2015, 160, 1125-1134. [CrossRef]

27. Errichelli, L.; Dini Modigliani, S.; Laneve, P.; Colantoni, A.; Legnini, I.; Capauto, D.; Rosa, A.; De Santis, R.; Scarfò, R.; Peruzzi, G.; et al. FUS affects circular RNA expression in murine embryonic stem cell-derived motor neurons. Nat. Commun. 2017, 8, 14741. [CrossRef]

28. Zhang, Y.; Xue, W.; Li, X.; Zhang, J.; Chen, S.; Zhang, J.-L.; Yang, L.; Chen, L.-L. The Biogenesis of Nascent Circular RNAs. Cell Rep. 2016, 15, 611-624. [CrossRef]

29. Wang, M.; Hou, J.; Müller-McNicoll, M.; Chen, W.; Schuman, E.M. Long and Repeat-Rich Intronic Sequences Favor Circular RNA Formation under Conditions of Reduced Spliceosome Activity. iScience 2019, 20, $237-247$. [CrossRef]

30. El Marabti, E.; Younis, I. The Cancer Spliceome: Reprograming of Alternative Splicing in Cancer. Front. Mol. Biosci. 2018, 5. [CrossRef]

31. Pellagatti, A.; Armstrong, R.N.; Steeples, V.; Sharma, E.; Repapi, E.; Singh, S.; Sanchi, A.; Radujkovic, A.; Horn, P.; Dolatshad, H.; et al. Impact of spliceosome mutations on RNA splicing in myelodysplasia: Dysregulated genes/pathways and clinical associations. Blood 2018, 132, 1225-1240. [CrossRef] [PubMed]

32. Suzuki, H.; Kumar, S.A.; Shuai, S.; Diaz-Navarro, A.; Gutierrez-Fernandez, A.; De Antonellis, P.; Cavalli, F.M.G.; Juraschka, K.; Farooq, H.; Shibahara, I.; et al. Recurrent noncoding U1 snRNA mutations drive cryptic splicing in SHH medulloblastoma. Nature 2019, 574, 707-711. [CrossRef]

33. Australian Institute of Health and Welfare. Cancer Data in Australia. Cat. no. CAN 122. Available online: https://www.aihw.gov.au/reports/cancer/cancer-data-in-australia (accessed on 15 November 2020).

34. Meliso, F.M.; Hubert, C.G.; Galante, P.A.F.; Penalva, L.O. RNA processing as an alternative route to attack glioblastoma. Hum. Genet. 2017, 136, 1129-1141. [CrossRef] [PubMed]

35. Chen, X.; Zhao, C.; Guo, B.; Zhao, Z.; Wang, H.; Fang, Z. Systematic Profiling of Alternative mRNA Splicing Signature for Predicting Glioblastoma Prognosis. Front. Oncol. 2019, 9. [CrossRef]

36. Sun, J.; Li, B.; Shu, C.; Ma, Q.; Wang, J. Functions and clinical significance of circular RNAs in glioma. Mol. Cancer 2020, 19, 34. [CrossRef]

37. Zhang, X.-O.; Dong, R.; Zhang, Y.; Zhang, J.-L.; Luo, Z.; Zhang, J.; Chen, L.-L.; Yang, L. Diverse alternative back-splicing and alternative splicing landscape of circular RNAs. Genome Res. 2016, 26, 1277-1287. [CrossRef]

38. Li, Y.; Chen, R.; Bowden, M.; Mo, F.; Lin, Y.-Y.; Gleave, M.; Collins, C.; Dong, X. Establishment of a neuroendocrine prostate cancer model driven by the RNA splicing factor SRRM4. Oncotarget 2017, 8 , 66878-66888. [CrossRef]

39. Li, Y.; Donmez, N.; Sahinalp, C.; Xie, N.; Wang, Y.; Xue, H.; Mo, F.; Beltran, H.; Gleave, M.; Wang, Y.; et al. SRRM4 Drives Neuroendocrine Transdifferentiation of Prostate Adenocarcinoma under Androgen Receptor Pathway Inhibition. Eur. Urol. 2017, 71, 68-78. [CrossRef]

40. Puchalski, R.B.; Shah, N.; Miller, J.; Dalley, R.; Nomura, S.R.; Yoon, J.-G.; Smith, K.A.; Lankerovich, M.; Bertagnolli, D.; Bickley, K.; et al. An anatomic transcriptional atlas of human glioblastoma. Science 2018, 360, 660-663. [CrossRef] 
41. Louis, D.N.; Perry, A.; Reifenberger, G.; von Deimling, A.; Figarella-Branger, D.; Cavenee, W.K.; Ohgaki, H.; Wiestler, O.D.; Kleihues, P.; Ellison, D.W. The 2016 World Health Organization Classification of Tumors of the Central Nervous System: A summary. Acta Neuropathol. (Berl.) 2016, 131, 803-820. [CrossRef]

42. Dobin, A.; Davis, C.A.; Schlesinger, F.; Drenkow, J.; Zaleski, C.; Jha, S.; Batut, P.; Chaisson, M.; Gingeras, T.R. STAR: Ultrafast universal RNA-seq aligner. Bioinforma. Oxf. Engl. 2013, 29, 15-21. [CrossRef] [PubMed]

43. Gao, Y.; Zhang, J.; Zhao, F. Circular RNA identification based on multiple seed matching. Brief. Bioinform. 2018, 19, 803-810. [CrossRef] [PubMed]

44. Quinlan, A.R.; Hall, I.M. BEDTools: A flexible suite of utilities for comparing genomic features. Bioinforma. Oxf. Engl. 2010, 26, 841-842. [CrossRef] [PubMed]

45. Travis, A.J.; Moody, J.; Helwak, A.; Tollervey, D.; Kudla, G. Hyb: A bioinformatics pipeline for the analysis of CLASH (crosslinking, ligation and sequencing of hybrids) data. Methods 2014, 65, 263-273. [CrossRef]

46. Shepelev, V.; Fedorov, A. Advances in the Exon-Intron Database (EID). Brief. Bioinform. 2006, 7, $178-185$. [CrossRef]

47. McCarthy, D.J.; Chen, Y.; Smyth, G.K. Differential expression analysis of multifactor RNA-Seq experiments with respect to biological variation. Nucleic Acids Res. 2012, 40, 4288-4297. [CrossRef]

48. Chen, Y.; Lun, A.T.L.; Smyth, G.K. From reads to genes to pathways: Differential expression analysis of RNA-Seq experiments using Rsubread and the edgeR quasi-likelihood pipeline. F1000Research 2016, 5, 1438. [CrossRef]

49. Anders, S.; Huber, W. Differential expression analysis for sequence count data. Genome Biol. 2010, 11, R106. [CrossRef]

50. Zwiener, I.; Frisch, B.; Binder, H. Transforming RNA-Seq data to improve the performance of prognostic gene signatures. PLoS ONE 2014, 9, e85150. [CrossRef]

51. Love, M.I.; Huber, W.; Anders, S. Moderated estimation of fold change and dispersion for RNA-seq data with DESeq2. Genome Biol. 2014, 15, 550. [CrossRef]

52. Blighe, K.; Rana, S.; Lewis, M. EnhancedVolcano: Publication-Ready Volcano Plots with Enhanced Colouring and Labeling. R Package Version 1.6.0. Available online: https://bioconductor.org/packages/devel/bioc/vignettes/ EnhancedVolcano/inst/doc/EnhancedVolcano.html (accessed on 1 September 2020).

53. Rokach, L.; Maimon, O. Clustering Methods; Data Mining and Knowledge Discovery Handbook; Maimon, O., Rokach, L., Eds.; Springer: Boston, MA, USA, 2005; pp. 321-352.

54. Conn, V.M.; Hugouvieux, V.; Nayak, A.; Conos, S.A.; Capovilla, G.; Cildir, G.; Jourdain, A.; Tergaonkar, V.; Schmid, M.; Zubieta, C.; et al. A circRNA from SEPALLATA3 regulates splicing of its cognate mRNA through R-loop formation. Nat. Plants 2017, 3, 17053. [CrossRef]

55. Conn, V.; Conn, S.J. SplintQuant: A method for accurately quantifying circular RNA transcript abundance without reverse transcription bias. RNA 2019, 25, 1202-1210. [CrossRef]

56. Gao, Y.; Wang, J.; Zhao, F. CIRI: An efficient and unbiased algorithm for de novo circular RNA identification. Genome Biol. 2015, 16, 4. [CrossRef]

57. Song, X.; Zhang, N.; Han, P.; Moon, B.-S.; Lai, R.K.; Wang, K.; Lu, W. Circular RNA profile in gliomas revealed by identification tool UROBORUS. Nucleic Acids Res. 2016, 44, e87. [CrossRef]

58. Memczak, S.; Jens, M.; Elefsinioti, A.; Torti, F.; Krueger, J.; Rybak, A.; Maier, L.; Mackowiak, S.D.; Gregersen, L.H.; Munschauer, M.; et al. Circular RNAs are a large class of animal RNAs with regulatory potency. Nature 2013, 495, 333-338. [CrossRef]

59. Shapiro, M.B.; Senapathy, P. RNA splice junctions of different classes of eukaryotes: Sequence statistics and functional implications in gene expression. Nucleic Acids Res. 1987, 15, 7155-7174. [CrossRef]

60. Tang, Z.; Li, C.; Kang, B.; Gao, G.; Li, C.; Zhang, Z. GEPIA: A web server for cancer and normal gene expression profiling and interactive analyses. Nucleic Acids Res. 2017, 45, W98-W102. [CrossRef]

61. Torres-Méndez, A.; Bonnal, S.; Marquez, Y.; Roth, J.; Iglesias, M.; Permanyer, J.; Almudí, I.; O’Hanlon, D.; Guitart, T.; Soller, M.; et al. A novel protein domain in an ancestral splicing factor drove the evolution of neural microexons. Nat. Ecol. Evol. 2019, 3, 691-701. [CrossRef]

62. Nakano, Y.; Wiechert, S.; Bánfi, B. Overlapping Activities of Two Neuronal Splicing Factors Switch the GABA Effect from Excitatory to Inhibitory by Regulating REST. Cell Rep. 2019, 27, 860-871. [CrossRef]

63. Nakano, Y.; Kelly, M.C.; Rehman, A.U.; Boger, E.T.; Morell, R.J.; Kelley, M.W.; Friedman, T.B.; Bánfi, B. Defects in the Alternative Splicing-Dependent Regulation of REST Cause Deafness. Cell 2018, 174, 536-548. [CrossRef] 
64. Uhlen, M.; Zhang, C.; Lee, S.; Sjöstedt, E.; Fagerberg, L.; Bidkhori, G.; Benfeitas, R.; Arif, M.; Liu, Z.; Edfors, F.; et al. A pathology atlas of the human cancer transcriptome. Science 2017, 357. [CrossRef] [PubMed]

65. Salzman, J.; Chen, R.E.; Olsen, M.N.; Wang, P.L.; Brown, P.O. Cell-type specific features of circular RNA expression. PLoS Genet. 2013, 9, e1003777. [CrossRef]

66. Gonatopoulos-Pournatzis, T.; Niibori, R.; Salter, E.W.; Weatheritt, R.J.; Tsang, B.; Farhangmehr, S.; Liang, X.; Braunschweig, U.; Roth, J.; Zhang, S.; et al. Autism-Misregulated eIF4G Microexons Control Synaptic Translation and Higher Order Cognitive Functions. Mol. Cell 2020, 77, 1176-1192. [CrossRef] [PubMed]

Publisher's Note: MDPI stays neutral with regard to jurisdictional claims in published maps and institutional affiliations.

(C) 2020 by the authors. Licensee MDPI, Basel, Switzerland. This article is an open access article distributed under the terms and conditions of the Creative Commons Attribution (CC BY) license (http://creativecommons.org/licenses/by/4.0/). 